\title{
Effects of Cognitive Behavior Therapy on Heart Rate Variability in Young Females with Constipation-predominant Irritable Bowel Syndrome: A Parallel-group Trial
}

\author{
Aelee Jang, ${ }^{1}$ Sun-Kyung Hwang, ${ }^{2 *}$ Nikhil S Padhye, ${ }^{1}$ and Janet C Meininger ${ }^{1}$ \\ ${ }^{1}$ School of Nursing, The University of Texas Health Science Center at Houston, Houston, TX, USA; and ${ }^{2}$ College of Nursing; Pusan National \\ University, Yangsan, Gyeongsangnam-do, Korea
}

\section{Background/Aims}

The relation between heart rate variability (HRV) as non-invasive biomarkers of autonomic function and cognitive behavior therapy (CBT) as non-pharmacological treatments has rarely been examined in patients with constipation-predominant irritable bowel syndrome (IBS-C). The purpose of this study is to evaluate the efficacy of an 8-week CBT intervention on HRV and IBS symptoms, and the correlation of changes in HRV with changes in IBS symptoms among young female nursing students with IBS-C.

\section{Methods}

This study consisted of an exploratory subgroup analysis of 43 participants with IBS-C who had been randomly assigned to receive either 8 weeks of CBT ( $n=23$ ) or general medical information (control, $n=20$ ). At baseline and 8, 16, and 24 weeks, participants completed a questionnaire assessing their gastrointestinal (GI) symptoms, anxiety, depression, and stress, and their HRV was measured via electrocardiography.

\section{Results}

At the 8-week follow-up, the high-frequency (HF) power was significantly higher, and the low-frequency (LF)/HF ratio was lower in the CBT group than in the control group ( $P<0.001$ for both), and the severity of $\mathrm{Gl}$ symptoms $(P=0.003)$, anxiety $(P<0.001)$, depression $(P<0.001)$, and stress $(P<0.001)$ was significantly lower in the CBT group than in the control group. Changes in the HF power were significantly and inversely associated with changes in GI symptoms, anxiety, depression, and stress at 16 and 24 weeks $(P<0.05$ for all; range of $r$ from -0.37 to -0.68$)$. Changes in the LF/HF ratio were also significantly and positively associated with changes in $\mathrm{Gl}$ symptoms, anxiety, depression, and stress at 16 and 24 weeks $(P<0.05$ for all; range of $r$ from 0.38 to 0.60$)$.

\section{Conclusions}

CBT was effective in managing symptoms in young IBS-C patients and the improvement of symptoms was sustained at 24 weeks following the completion of CBT. Furthermore, indirect measurement of autonomic function using HRV may be a useful objective parameter for assessing response to CBT in young IBS-C patients.

(J Neurogastroenterol Motil 2017;23:435-445)

\section{Key Words}

Autonomic nervous system; Cognitive therapy; Female; Irritable bowel syndrome; Young adult

Received: February 13, 2017 Revised: April 14, 2017 Accepted: April 28, 2017

(c) This is an Open Access article distributed under the terms of the Creative Commons Attribution Non-Commercial License (http://creativecommons. org/licenses/by-nc/4.0) which permits unrestricted non-commercial use, distribution, and reproduction in any medium, provided the original work is properly cited.

${ }^{*}$ Correspondence: Sun-Kyung Hwang, PhD College of Nursing, Pusan National University, 49 Busandaehak-ro, Mulgeum-eup, Yangsan, Gyeongsangnam-do 50612, Korea Tel: +82-51-510-8340, Fax: +82-51-510-8308, E-mail: skhwang@pusan.ac.kr 


\section{Introduction}

Irritable bowel syndrome (IBS) is a common functional gastrointestinal (GI) disorder affecting $10 \%$ to $22 \%$ of the adult population. ${ }^{1-4}$ IBS is subcategorized by the predominant stool pattern as constipation-predominant (IBS-C), diarrhea-predominant (IBS-D), mixed stool pattern (IBS-M), and unclassifiable (IBS$\mathrm{U})$. Among the subtypes, IBS-C is the most prevalent, accounting for at least one-third to $58 \%$ of all IBS, particularly in females and younger individuals. ${ }^{5-9}$ IBS interferes with social function, decreases quality of life, and increases health care costs. ${ }^{10} \mathrm{~A}$ systematic review on IBS indicated that the direct annual cost of managing IBS ranges from approximately $\$ 1600$ to $\$ 7500$ per person. ${ }^{1,10}$

IBS has been attributed to GI sensory-motor dysfunction, such as intestinal dysmotility, visceral hypersensitivity, intestinal bacterial overgrowth, inflammation, food sensitivity or intolerance, genetic factors, and psychosocial dysfunction. ${ }^{3,11-13}$ Recent evidence suggests that alterations in the brain-gut axis play an important role in IBS, ${ }^{14,15}$ including altered sympathetic and parasympathetic activities of the autonomic nervous system (ANS). ${ }^{16}$ Similarly, patients with IBS are known to have a higher occurrence of mood disturbances, anxiety, depression, somatization disorders, and psychological distress, ${ }^{15,17}$ and the central nervous system (CNS) is believed to be involved in the concomitance of these conditions. ${ }^{15,18,19}$

Heart rate variability (HRV) is a noninvasive measure of ANS activities. Power spectral analysis of HRV allows the assessment of predominance in sympathetic or parasympathetic activities. Typically, low-frequency (LF) bands are associated with sympathetic activity, whereas high-frequency (HF) bands are associated with a parasympathetic/vagal tone. The vagal tone and balance of sympathetic and parasympathetic activities are the indices of the significance of HRV. ${ }^{18,20,21}$ In a previous study, IBS-C patients had lower $\mathrm{HF}$ power and a higher $\mathrm{LF} / \mathrm{HF}$ ratio than healthy controls, which suggests parasympathetic dysfunction and sympathetic overactivity. ${ }^{3,5}$

Non-pharmacologic treatments, such as cognitive behavior therapy (CBT), have been effective in improving somatic symptoms of IBS. ${ }^{15,22,23}$ The mechanism by which CBT improves somatic symptoms is related to the regulation of brain-gut axis function. ${ }^{15,22,24} \mathrm{HRV}$ is an objective, noninvasive biomarker that reflects current ANS function correlated with IBS symptoms. Thus, HRV may supplement clinical decisions in IBS patients who undergo CBT. But, it is unclear whether HRV changes related to CBT correlate with changes in bowel symptoms or psychosocial responses in IBS patients. Moreover, sustained effects of CBT have not been adequately tested in IBS patients. ${ }^{23}$

Therefore, the specific aims of this study are to evaluate (1) the responses of HRV, GI symptoms, anxiety, depression, and stress to an 8-week CBT intervention and (2) the associations of changes in HRV responses with changes in GI symptoms, anxiety, depression, and stress in young female nursing students with IBS-C. We hypothesized that the CBT group would have increased $\mathrm{HF}$ power and decreased $\mathrm{LF} / \mathrm{HF}$ ratio correlating with improvements in GI symptoms, anxiety, depression, and stress, whereas the control group would have no significant changes. We further hypothesized that these stated changes would be observed immediately after CBT and sustained at 16 and 24 weeks following the completion of CBT.

This was an exploratory subgroup analysis of a larger study in which the efficacy of a CBT intervention ( 80 minutes per week for 8 weeks) on bowel symptom severity, dysfunctional attitudes, and IBS-quality of life was examined in young female nursing students with all types of IBS. ${ }^{24}$ Young females nursing students were selected because of the high prevalence of IBS in this population. ${ }^{24}$ They are also noted to be similar lifestyles such as school lives and food patterns. ${ }^{24}$ IBS-C patients was focused on due to a unique pattern of HRV and GI symptoms. ${ }^{5}$ IBS-C patients are known experience more severe upper GI symptoms and lower GI bloating than those with other subtypes of IBS, suggesting greater vagal mediation of afferent signals. ${ }^{8,19}$

\section{Materials and Methods}

\section{Study Design}

This was an exploratory subgroup analysis of a randomized controlled trial study in which the efficacy of an 8-week CBT intervention was examined in young female nursing students with all types of IBS. ${ }^{24}$ Data were collected 4 times from both the CBT and control groups: prior to CBT (baseline) and after 8, 16, and 24 weeks.

\section{Participants}

Participants were recruited from 4 colleges of nursing in the Republic of Korea. Participants were included if they were diagnosed as IBS-C by a gastroenterologist based on the questionnaire according to the Rome III criteria. ${ }^{25}$ After giving written informed consent, the participants were screened by a physician assistant to evaluate their vital signs and baseline physical condition; were at 


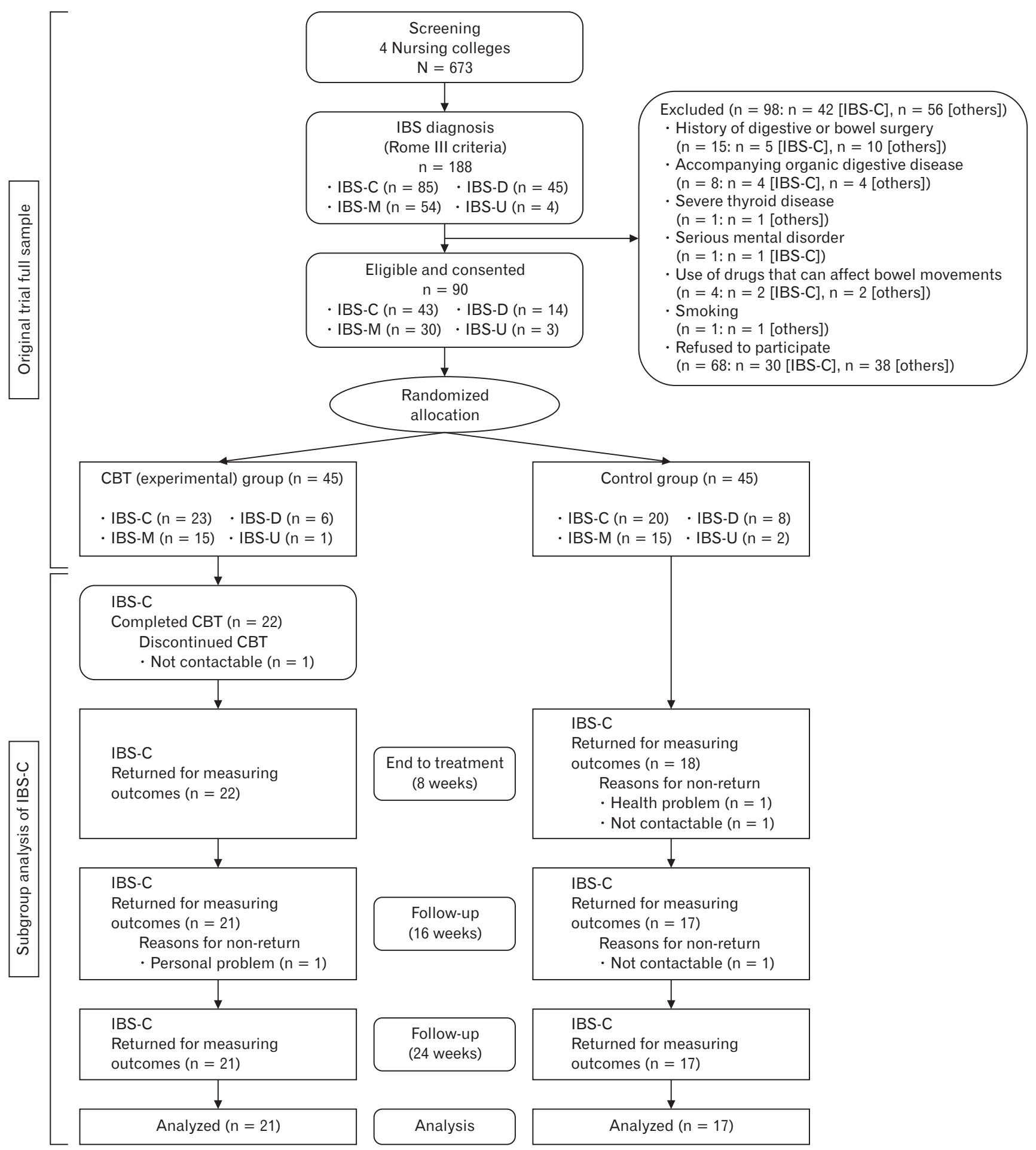

Figure. CONSORT flow diagram identifies the number of exploratory subgroup enrollment in each phase. IBS, irritable bowel syndrome; IBS-C, constipation-predominant IBS; IBS-D, diarrhea-predominant IBS; IBS-M, mixed stool pattern IBS; IBS-U, unclassifiable IBS; CBT, cognitive behavior therapy. 
least 18 years old; were female; and were enrolled as a nursing student. Individuals were excluded if they had a history of digestive or bowel surgery that may have caused similar symptoms; had an accompanying organic digestive disease that had the potential to affect their symptoms (eg, inflammatory bowel disease, lactose malabsorption, celiac disease, obstructive bowel disease, etc); had severe thyroid disease; had a serious mental disorder; used any drug that can affect bowel movements (eg, prokinetics, antispasmodics, digestive stimulants, antidiarrheal agents, antibiotics, antihistamines, laxatives, etc); or smoked. ${ }^{24}$ Participants were assigned randomly to the CBT or control group using a random number generator (https:// www.randomizer.org/). When all study participants gave written consent, they were not informed of their group assignment and were told that those assigned to the control group would be offered the CBT program after the completion of the study. Of the 85 participants with IBS-C, 42 were not eligible, and 43 participants were randomly assigned to either the CBT group $(n=23)$ or control group $(\mathrm{n}=20)$ (Figure). Two participants from the CBT group and 3 from the control group withdrew from the study because of personal problems $(n=1)$, health problems $(n=1)$, and loss of contact $(\mathrm{n}=3)$, resulting in 38 participants having completed the study.

\section{Intervention}

The CBT intervention consisted of 8 weekly group sessions of 80 minutes per session, with 60 minutes of thematic training and 20 minutes of relaxation training. Major topics included establishing a therapeutic relationship and setting a therapeutic goal (session 1), education on IBS related to CBT (session 2), training in cognitive restructuring (sessions 3-5), training in effective coping (sessions 6 and 7), and training in enhancing positive emotion (session 8). ${ }^{24}$ CBT was conducted in a group of 4-6 participants by the same psychotherapist. The control group received general information about IBS (eg, overview, cause, symptoms, treatments, and diet) and other usual care for 50 minutes during the first week. They were also assigned to a group of 4-6 people and interviewed by the investigator about their GI symptoms. All data were collected at the designated time points from all participants in small groups of 4-6 by one trained researcher who was unaware of group assignment. Data were collected by administering questionnaires and assessing HRV at baseline and 8, 16, and 24 weeks.

\section{Sample Size}

Sample size for the larger study was determined on the basis of primary endpoints of 3 outcomes for which Bonferroni's corrected $\alpha$ value ( $\alpha=0.05 / 3=0.0167$ ) was used. Therefore, we calculated that the sample size required for the larger study was $\mathrm{N}=80$, with previously reported ${ }^{24}$ effect sizes of $f=0.45, \alpha=0.0167$, and $\beta=$ 0.2 for repeated-measures analysis of variance and with an assumption of $10 \%$ attrition. ${ }^{26}$ For this subgroup analysis of participants with IBS-C ( $\mathrm{n}=43)$, we estimated that power would range from 0.57 to 0.98 when the unknown effect size varied between $\mathrm{f}=0.45$ and 0.75 for the 2 primary HRV outcomes (adjusted $\alpha=0.05$ ).

\section{Ethical Considerations}

This study was approved by the Institutional Review Board of Pusan National University Hospital and was registered with the Clinical Research Information Service of Korea (KCT0000768: member of the WHO International Clinical Trials Registry Platform: https://cris.nih.go.kr/cris/index.jsp). Participants received a small gift package to compensate for their time and effort in the study.

\section{Primary Outcome}

HRV was measured using a QECG-3 monitoring system (Laxtha Inc, Daejeon, Korea). HRV is a noninvasive method that provides quantitative evaluation of sympathovagal interactions modulating cardiac function. ${ }^{27,28} \mathrm{HRV}$ is mediated by the combined effects of both the parasympathetic (vagal) and sympathetic nervous systems on the heart rate. Since the parasympathetic and sympathetic effects on the cardiac cycle cause fluctuations at different frequencies, they can be separately extracted from power spectral analysis of the HRV signal. HRV measures were derived from the electrocardiogram (ECG) signals. After participants rested for 10 minutes in a quiet environment, ECG electrodes were attached to both wrists and ankles, and ECG was recorded for 10 minutes in a comfortable sitting position. To control for unintended influence on HRV, participants were asked not to drink coffee or alcohol or exercise for an hour before the HRV measurement. This study used frequency domain analysis of HRV, known as power spectral analysis. ECG data were fed to a computerized data collection system to interface with data analysis software (TeleScan Ver.2.8; Laxtha Inc). Digitized ECG signals were used to identify R-wave peaks, and beat-to-beat heart rate intervals were calculated (ie, R-R intervals).

The R-R intervals were subjected to spectral analysis that yielded HRV measures in 2 main frequency bands: the HF band (0.15$0.4 \mathrm{~Hz}$ ) indicating cardiac vagal tone and the LF band (0.04-0.15 $\mathrm{Hz}$ ) largely indicating sympathetic activity. Power in the LF, however, is also thought to include some vagal influences ${ }^{27,29}$; thus, the interpretation of the LF parameter may not be as pure. However, 
the ratio of the $\mathrm{LF}$ and $\mathrm{HF}$ components, the $\mathrm{LF} / \mathrm{HF}$ ratio, typically represents an index of sympathetic-to-parasympathetic balance, with higher values indicating greater sympathetic dominance. ${ }^{27,28}$

\section{Secondary Outcomes}

GI symptoms were measured using a version of the GI Symptom Rating Scale (GSRS) ${ }^{30,31}$ supplemented with questions about the Rome I criteria for IBS. The GSRS-IBS comprises of 13 items to assess the severity of GI symptoms, including pain, bloating, diarrhea, constipation, and satiety. Each item is scored from $1=$ no discomfort at all to $7=$ very severe discomfort, leading to a total score ranging from 13 to 91 . Higher scores indicate a higher severity of GI symptoms. The GSRS-IBS Cronbach's $\alpha$ in this study was 0.91 .

Anxiety and depression were assessed using the 14-item selfadministered Hospital Anxiety and Depression Scale (HADS). ${ }^{32,33}$ Each subscale (anxiety and depression) includes 7 items that are rated on a Likert scale of $0=$ not at all to $3=$ definitely/most of the time, with a potential total score on each subscale ranging from 0 to 21. Higher scores indicate higher levels of anxiety and depression. Cronbach's $\alpha$ for the anxiety and depression subscales in this study were 0.83 and 0.80 .

Stress was measured using the Global Assessment of Recent Stress (GARS), which evaluates the severity of recent stressors during the 1 week preceding its administration. ${ }^{34,35}$ The instrument comprises of 7 individual items and one overall item that relate to respondents' job, work, pressure from personal relations, changes in personal relations, disease, financial status, daily life, and changes in daily life that are perceived as general stress. The GARS is based on a 10 -point scale $(0=$ no stress to $9=$ severely stressful state $)$, with a total score ranging from 0 to 72 . Higher scores indicate higher levels of stress. The GARS Cronbach's $\alpha$ in this study was 0.91 .

Demographic information was collected via a background questionnaire. Items included age, body mass index, education level, residential status, exercise, and regularity of meals.

\section{Statistical Methods}

Descriptive data were summarized by frequency and percentage for categorical data and mean \pm standard deviation for continuous data. Baseline group differences were tested using Chi-square tests for categorical data and independent-sample $t$ tests for numeric data. To correct skewed distributions, logarithmic transformations were applied to the primary spectral analysis-based $\mathrm{HRV}$ measures used in this study (HF, LF/HF ratio). For clarity, descriptive statistical summaries of the $\mathrm{HRV}$ measures (HF $\mathrm{LF} / \mathrm{HF}$ ratio) are reported in the original metrics, ie, without logarithmic transformation. The longitudinally measured outcomes were analyzed with linear mixed effects models. The linear mixed models for HF and $\mathrm{LF} / \mathrm{HF}$ ratio contained group (CBT and control) and time as fixed effects, a random intercept to account for within-subject correlation, and baseline values of corresponding outcome (HF or LF/ HF ratio) and GI symptoms, anxiety, depression, and stress level at baseline as fixed-effect covariates. The coefficient for the group x time interaction measured the difference in the improvement of an outcome between the CBT and control groups. The linear mixed models for GI symptoms, anxiety, depression, and stress were similar, and controlled for baseline values of each outcome. Model diagnostics satisfied linearity and homogeneity assumptions. The estimated marginal means of outcomes at 8, 16, and 24 weeks were derived from the linear mixed models. Simple contrasts were used to compare the outcomes between groups at each time point, and a sequential Sidak correction was used for multiple comparisons.

Bivariate Pearson correlations were used to test the associations between changes in HRV responses and changes in GI symptoms, anxiety, depression, and stress levels. Changes in all outcomes were approximately normally distributed. All statistical analyses were performed using SPSS version 22.0 (IBM Corp, Armonk, NY USA), and the level of significance was set at $\alpha=0.05$.

\section{Results}

\section{Characteristics of Participants}

The mean age of participants $(n=43)$ was $21.4 \pm 2.1$ years, and the mean body mass index was $19.9 \pm 1.8 \mathrm{~kg} / \mathrm{m}^{2}$. The majority (60.5\%) resided in their own house, and $46.5 \%$ ate regular meals about 4-6 days per week (Table 1). The CBT and control groups did not differ in any demographic factors or baseline measures of the study variables.

\section{High-frequency and Low-frequency/High-frequency Ratio Responses to Cognitive Behavior Therapy}

Controlling for baseline values of $\mathrm{HF}$ or $\mathrm{LF} / \mathrm{HF}$ ratios and covariates (baseline of gastrointestinal symptom, anxiety, depression, and stress), linear mixed effects models showed that group x time interactions were significant in $\mathrm{HF}$ and $\mathrm{LF} / \mathrm{HF}$ ratio response $(P<$ 0.001 for both; Table 2$)$. The significant main effects of time $(P=$ 0.001 and $P<0.001$, respectively) indicated that the differences persisted across all follow-up times. This finding was confirmed in the contrasts at each follow-up, which showed significant differ- 
Table 1. Demographic and Clinical Characteristics of Participants

\begin{tabular}{|c|c|c|c|c|c|c|}
\hline \multirow{2}{*}{ Characteristics } & \multirow{2}{*}{ Categories } & Total $(n=43)$ & $\operatorname{CBT}(\mathrm{n}=23)$ & Cont $(n=20)$ & \multirow{2}{*}{$\chi^{2} / t$} & \multirow{2}{*}{$P$} \\
\hline & & \multicolumn{3}{|c|}{ Mean $\pm \mathrm{SD}$ or participants $(\%)$} & & \\
\hline Age (yr) & & $21.4 \pm 2.1$ & $21.6 \pm 1.8$ & $21.2 \pm 2.4$ & 0.64 & 0.523 \\
\hline Body mass index $\left(\mathrm{kg} / \mathrm{m}^{2}\right)$ & & $19.9 \pm 1.8$ & $19.7 \pm 1.6$ & $20.2 \pm 2.0$ & -0.79 & 0.433 \\
\hline \multirow{4}{*}{ Education level } & Freshman & $11(25.6)$ & $4(17.4)$ & $7(35.0)$ & 3.49 & 0.322 \\
\hline & Sophomore & $6(14.0)$ & $5(21.7)$ & $1(5.0)$ & & \\
\hline & Junior & $6(14.0)$ & $3(13.0)$ & $3(15.0)$ & & \\
\hline & Senior & $20(46.4)$ & $11(47.9)$ & $9(45.0)$ & & \\
\hline \multirow[t]{4}{*}{ Residential status } & Own house & $26(60.5)$ & $14(60.9)$ & $12(60.0)$ & 2.29 & 0.515 \\
\hline & Dormitory & $12(27.9)$ & $5(21.7)$ & $7(35.0)$ & & \\
\hline & Self-boarding & $4(9.3)$ & $3(13.0)$ & $1(5.0)$ & & \\
\hline & Others & $1(2.3)$ & $1(4.3)$ & $0(0.0)$ & & \\
\hline \multirow[t]{2}{*}{ Exercise } & Irregularly & $24(55.8)$ & $13(56.5)$ & $11(55.0)$ & 0.01 & $>0.99$ \\
\hline & Not at all & $19(44.2)$ & $10(43.5)$ & $9(45.0)$ & & \\
\hline \multirow[t]{4}{*}{ Regularity of meals (per wk) } & Everyday & $1(2.3)$ & $0(0.0)$ & $1(5.0)$ & 1.94 & 0.584 \\
\hline & 4-6 days & $20(46.5)$ & $10(43.5)$ & $10(50.0)$ & & \\
\hline & 1-3 days & $14(32.6)$ & $9(39.1)$ & $5(25.0)$ & & \\
\hline & Not at all & $8(18.6)$ & $4(17.4)$ & $4(20.0)$ & & \\
\hline Gastrointestinal symptoms & & $33.9 \pm 12.8$ & $34.1 \pm 8.7$ & $33.8 \pm 16.6$ & 0.08 & 0.936 \\
\hline Anxiety & & $8.7 \pm 3.1$ & $8.7 \pm 2.4$ & $8.6 \pm 3.7$ & 0.10 & 0.920 \\
\hline Depression & & $7.3 \pm 2.6$ & $7.3 \pm 2.7$ & $7.4 \pm 2.6$ & -0.11 & 0.912 \\
\hline Stress & & $27.9 \pm 14.3$ & $28.7 \pm 13.4$ & $27.0 \pm 15.5$ & 0.40 & 0.695 \\
\hline \multirow[t]{2}{*}{ Heart rate variability } & $\mathrm{HF}$ & $308.0 \pm 229.8$ & $305.4 \pm 198.7$ & $310.9 \pm 266.3$ & -0.08 & 0.940 \\
\hline & $\mathrm{LF} / \mathrm{HF}$ & $2.04 \pm 1.21$ & $2.03 \pm 1.08$ & $2.05 \pm 1.38$ & -0.06 & 0.953 \\
\hline
\end{tabular}

CBT, cognitive behavior therapy group; Cont, control group; HF, high-frequency; LF/HF, low-frequency/high-frequency.

Table 2. Estimated Marginal Mean for Linear Mixed Models of High-frequency and Low-frequency/High-frequency Ratio Responses over Time in Cognitive Behavior Therapy and Control Groups

\begin{tabular}{|c|c|c|c|c|c|c|}
\hline \multirow{2}{*}{ Variables } & \multicolumn{3}{|c|}{ Estimated marginal mean (95\% CI: low-high) } & \multirow{2}{*}{$\begin{array}{c}\text { Group } \\
P^{a}\end{array}$} & \multirow{2}{*}{$\begin{array}{c}\text { Time } \\
P^{\mathrm{a}}\end{array}$} & \multirow{2}{*}{$\frac{\text { Gr x Time }}{P^{\mathrm{a}}}$} \\
\hline & $8 \mathrm{wk}$ & 16 wk & $24 \mathrm{wk}$ & & & \\
\hline $\mathrm{HF}$ & & & & $<0.001$ & 0.001 & $<0.001$ \\
\hline CBT group & $282.9(245.2-326.4)$ & $323.4(284.9-366.9)$ & $384.1(337.6-437.0)$ & & & \\
\hline Control group & $217.9(185.9-255.2)$ & $232.5(201.9-267.5)$ & $221.2(191.5-255.2)$ & & & \\
\hline$P^{\mathrm{b}}$ & 0.017 & 0.001 & $<0.001$ & & & \\
\hline $\mathrm{LF} / \mathrm{HF}$ & & & & $<0.001$ & $<0.001$ & $<0.001$ \\
\hline CBT group & $1.50(1.35-1.67)$ & $1.15(1.05-1.24)$ & $1.05(1.05-1.15)$ & & & \\
\hline Control group & $1.91(1.70-2.15)$ & $1.93(1.76-2.11)$ & $1.95(1.76-2.17)$ & & & \\
\hline$P^{\mathrm{b}}$ & 0.003 & $<0.001$ & $<0.001$ & & & \\
\hline
\end{tabular}

Exponentiated marginal means shown of model using log-transformed high-frequency (HF) or low-frequency (LF)/HF ratio.

$P^{\mathrm{a}}$ and $P^{\mathrm{b}}$ indicate the significance values of predictors in the linear mixed models controlling for baseline values of each outcome (HF or LF/HF) and covariates (baseline of gastrointestinal symptoms, anxiety, depression, and stress).

CBT, cognitive behavior therapy.

ences between the CBT and control groups in $\operatorname{HF}(P=0.017$ at 8 weeks, $P=0.001$ at 16 weeks, and $P<0.001$ at 24 weeks) and $\mathrm{LF} / \mathrm{HF}$ ratio $(P=0.003$ at 8 weeks and $P<0.001$ at 16 and 24 weeks). The estimated marginal mean of $\mathrm{HF}$ increased in the
CBT group at follow-up but was approximately unchanged in the control group. The estimated marginal mean for the $\mathrm{LF} / \mathrm{HF}$ ratio decreased in the CBT group but was approximately unchanged in the control group. 
Table 3. Estimated Marginal Mean for Linear Mixed Models of Gastrointestinal Symptoms, Anxiety, Depression, and Stress Responses over Time in Cognitive Behavior Therapy and Control Groups

\begin{tabular}{|c|c|c|c|c|c|c|}
\hline \multirow{2}{*}{ Variables } & \multicolumn{3}{|c|}{ Estimated marginal mean (SE) } & \multirow{2}{*}{$\begin{array}{c}\text { Group } \\
P^{a}\end{array}$} & \multirow{2}{*}{$\begin{array}{c}\text { Time } \\
P^{\mathrm{a}}\end{array}$} & \multirow{2}{*}{$\begin{array}{c}\text { Gr x Time } \\
P^{\mathrm{a}}\end{array}$} \\
\hline & $8 \mathrm{wk}$ & 16 wk & $24 \mathrm{wk}$ & & & \\
\hline GI symptoms & & & & $<0.001$ & 0.008 & 0.003 \\
\hline CBT group & $24.3(1.23)$ & $20.1(1.00)$ & $18.9(1.18)$ & & & \\
\hline Control group & $34.3(1.37)$ & $34.2(1.11)$ & $35.0(1.31)$ & & & \\
\hline$P^{\mathrm{b}}$ & $<0.001$ & $<0.001$ & $<0.001$ & & & \\
\hline Anxiety & & & & $<0.001$ & 0.013 & $<0.001$ \\
\hline CBT group & $5.98(0.33)$ & $4.55(0.29)$ & $4.22(0.26)$ & & & \\
\hline Control group & $8.84(0.36)$ & $8.77(0.32)$ & $9.42(0.29)$ & & & \\
\hline$P^{\mathrm{b}}$ & $<0.001$ & $<0.001$ & $<0.001$ & & & \\
\hline Depression & & & & $<0.001$ & 0.019 & $<0.001$ \\
\hline CBT group & $5.44(0.31)$ & $4.19(0.30)$ & $3.71(0.22)$ & & & \\
\hline Control group & $7.65(0.35)$ & $7.92(0.33)$ & $8.16(0.25)$ & & & \\
\hline$P^{\mathrm{b}}$ & $<0.001$ & $<0.001$ & $<0.001$ & & & \\
\hline Stress & & & & $<0.001$ & 0.255 & $<0.001$ \\
\hline CBT group & $19.8(1.37)$ & $15.7(1.21)$ & $14.7(1.11)$ & & & \\
\hline Control group & $28.3(1.52)$ & $31.6(1.34)$ & $31.3(1.23)$ & & & \\
\hline$P^{\mathrm{b}}$ & $<0.001$ & $<0.001$ & $<0.001$ & & & \\
\hline
\end{tabular}

$P^{\mathrm{a}}$ and $P^{\mathrm{b}}$ indicate the significance values of predictors in the linear mixed models controlling for baseline values of each outcome (gastrointestinal symptoms, anxiety, depression, or stress).

CBT, cognitive behavior therapy; GI, gastrointestinal; SE, standard error.

\section{Gastrointestinal Symptoms, Anxiety, Depression, and Stress Responses to Cognitive Behavior Therapy}

Controlling for baseline values of GI symptoms, anxiety, depression, or stress, linear mixed effects models showed that group x time interactions were significant in GI symptoms, anxiety, depression, and stress, while the main effect of time was significant in all outcomes except stress (Table 3). GI symptoms, anxiety, depression, and stress were significantly different between the $\mathrm{CBT}$ and control groups at each follow-up ( $P<0.001$ at 8,16 , and 24 weeks). The estimated marginal means for GI symptoms, anxiety, depression, and stress decreased in the CBT group but were approximately unchanged in the control group.

\section{Associations of Changes in High-frequency and Low-frequency/High-frequency Ratio Responses with Changes in Gastrointestinal Symptoms, Anxiety, Depression, and Stress}

Correlation coefficients summarized in Table 4 show that changes in the HF power were significantly and inversely associated with changes in GI symptoms, anxiety, depression, and stress at 16 and 24 weeks with a range of $r=-0.37$ to $r=-0.68(P<0.05$ for all). Changes in the LF/HF ratios were significantly and positively associated with changes in GI symptoms, anxiety, depression, and stress at 16 and 24 weeks with a range of $r=0.38$ to $r=0.60$ $(P<0.05$ for all). At 8 weeks, changes in the $\mathrm{LF} / \mathrm{HF}$ ratios were significantly associated only with changes in depression $(r=0.44$, $P<0.01)$.

\section{Discussion}

The aim of this study was to evaluate the responses of HRV, GI symptoms, anxiety, depression, and stress to an 8-week CBT, and to evaluate the correlation of changes in $\mathrm{HRV}$ with changes in GI symptoms, anxiety, depression, and stress among young female nursing students with IBS-C over a 24-week follow-up period. We found that $\mathrm{CBT}$ resulted in a significant increase in $\mathrm{HF}$ and significant decrease in the LF/HF ratio, GI symptoms, anxiety, depression, and stress. Furthermore, the magnitude of changes in $\mathrm{HF}$ and the $\mathrm{LF} / \mathrm{HF}$ ratios were significantly associated with the magnitude of changes in GI symptoms, anxiety, depression, and stress in this study sample. Our results suggest that indirect measurement of ANS activity using HRV may be a useful objective parameter for assessing the response to CBT in young patients with IBS-C. To our knowledge, this is the first report with regard to changes in HRV patterns after CBT in IBS patients.

Because of its association with ANS dysfunction, HRV has 
Table 4. Associations of Change Scores in High-frequency and Low-frequency/High-frequency Ratio Responses with Change Scores of Gastrointestinal Symptoms, Anxiety, Depression, and Stress

\begin{tabular}{|c|c|c|c|c|c|c|}
\hline \multirow{2}{*}{ Variables } & \multicolumn{3}{|c|}{$\mathrm{HF}$} & \multicolumn{3}{|c|}{$\mathrm{LF} / \mathrm{HF}$} \\
\hline & Baseline-8 wk & Baseline-16 wk & Baseline-24 wk & Baseline-8 wk & Baseline-16 wk & Baseline-24 wk \\
\hline \multicolumn{7}{|l|}{ GI Symptoms } \\
\hline Baseline-8 wk $(\mathrm{n}=40)$ & -0.21 & $-0.42^{\mathrm{a}}$ & $-0.64^{\mathrm{a}}$ & 0.13 & $0.34^{\mathrm{b}}$ & $0.36^{\mathrm{b}}$ \\
\hline Baseline-16 wk $(\mathrm{n}=38)$ & -0.26 & $-0.55^{\mathrm{a}}$ & $-0.76^{\mathrm{a}}$ & 0.22 & $0.55^{\mathrm{a}}$ & $0.54^{\mathrm{a}}$ \\
\hline Baseline-24 wk $(\mathrm{n}=38)$ & -0.22 & $-0.47^{\mathrm{a}}$ & $-0.68^{\mathrm{a}}$ & 0.05 & $0.36^{\mathrm{b}}$ & $0.38^{\mathrm{b}}$ \\
\hline \multicolumn{7}{|l|}{ Anxiety } \\
\hline Baseline-8 wk $(\mathrm{n}=40)$ & -0.22 & $-0.32^{\mathrm{b}}$ & $-0.39^{\mathrm{b}}$ & 0.18 & 0.22 & 0.25 \\
\hline Baseline-16 wk $(\mathrm{n}=38)$ & $-0.34^{\mathrm{b}}$ & $-0.42^{\mathrm{a}}$ & $-0.54^{\mathrm{a}}$ & $0.38^{\mathrm{b}}$ & $0.60^{\mathrm{a}}$ & $0.59^{\mathrm{a}}$ \\
\hline Baseline-24 wk $(\mathrm{n}=38)$ & $-0.31^{\mathrm{b}}$ & $-0.48^{\mathrm{a}}$ & $-0.65^{\mathrm{a}}$ & $0.38^{\mathrm{b}}$ & $0.58^{\mathrm{a}}$ & $0.60^{\mathrm{a}}$ \\
\hline \multicolumn{7}{|l|}{ Depression } \\
\hline Baseline-8 wk $(\mathrm{n}=40)$ & -0.28 & -0.27 & -0.32 & $0.44^{\mathrm{a}}$ & $0.38^{\mathrm{b}}$ & $0.38^{\mathrm{b}}$ \\
\hline Baseline-16 wk $(\mathrm{n}=38)$ & $-0.46^{\mathrm{a}}$ & $-0.37^{\mathrm{b}}$ & $-0.49^{\mathrm{a}}$ & $0.38^{\mathrm{b}}$ & $0.49^{\mathrm{a}}$ & $0.46^{\mathrm{a}}$ \\
\hline Baseline-24 wk $(\mathrm{n}=38)$ & $-0.46^{\mathrm{a}}$ & $-0.41^{\mathrm{b}}$ & $-0.56^{\mathrm{a}}$ & 0.29 & $0.54^{\mathrm{a}}$ & $0.51^{\mathrm{a}}$ \\
\hline \multicolumn{7}{|l|}{ Stress } \\
\hline Baseline-8 wk $(\mathrm{n}=40)$ & 0.09 & -0.27 & $-0.41^{\mathrm{b}}$ & 0.25 & 0.31 & 0.31 \\
\hline Baseline-16 wk $(\mathrm{n}=38)$ & -0.23 & $-0.46^{\mathrm{a}}$ & $-0.55^{\mathrm{a}}$ & 0.23 & $0.46^{\mathrm{a}}$ & $0.46^{\mathrm{a}}$ \\
\hline Baseline-24 wk $(\mathrm{n}=38)$ & -0.24 & $-0.44^{\mathrm{a}}$ & $-0.54^{\mathrm{a}}$ & 0.21 & $0.41^{\mathrm{b}}$ & $0.44^{\mathrm{a}}$ \\
\hline
\end{tabular}

HF, high-frequency; LF/HF, low-frequency/high-frequency; GI, gastrointestinal.

${ }^{a} P<0.01,{ }^{b} P<0.05$.

been evaluated in IBS patients. Mazur et $\mathrm{al}^{3}$ showed that HF power was significantly lower and the LF/HF ratio was significantly higher in IBS-C patients than in a non-IBS control group. Furthermore, among women with severe gut pain, Cain et $\mathrm{al}^{36}$ found that $\mathrm{HF}$ power was significantly lower and the $\mathrm{LF} / \mathrm{HF}$ ratio was significantly higher in IBS-C patients than in IBS-D patients. Constipation is a major symptom in patients with decreased parasympathetic activity (indicated by lower HF power) and imbalanced vagal/sympathetic activity (indicated by higher $\mathrm{LF} / \mathrm{HF}$ ratio). ${ }^{5,21,37}$ Low vagal activity may be associated with increased norepinephrine levels, ${ }^{36}$ heightened esophageal acid sensitivity, ${ }^{21}$ blockage of acetylcholine from enteric nerves, ${ }^{5}$ reduced release of serotonin in plasma in the enterochromaffin cells, ${ }^{37}$ visceral hypersensitivity, ${ }^{5,36}$ inhibitory motor neurons in the GI tract, ${ }^{5,37}$ and changes in the frequency of high-amplitude contractions in the colon. ${ }^{21,37}$ In our study, all IBS$\mathrm{C}$ patients had relatively lower $\mathrm{HF}$ and higher $\mathrm{LF} / \mathrm{HF}$ ratios at baseline than after CBT, which was consistent with the expected relationship between low vagal tone and IBS-C as reported in previous studies. $^{3,5,21,36}$

Our results demonstrate that $\mathrm{CBT}$ induced significant changes in HRV by increasing the $\mathrm{HF}$ and decreasing the $\mathrm{LF} / \mathrm{HF}$ ratio. The HRV changes after CBT indirectly indicate increased vagal tone and decreased sympathetic power, and the CBT group also had greater improvements in GI symptoms than the control group.
Moreover, HRV changes throughout follow-up positively correlated with IBS symptoms. In a previous study that investigated the association between baseline HRV and changes in GI symptoms after a cognitively focused behavioral intervention in patients with IBS, IBS patients with lower $\mathrm{HF}$ and a higher $\mathrm{LF} / \mathrm{HF}$ ratio at baseline exhibited less benefit from the intervention with regard to GI symptoms than those with higher $\mathrm{HF}$ and lower LF/HF at baseline. ${ }^{23}$ The authors of that study suggested that IBS patients with lower $\mathrm{HF}$ and higher $\mathrm{LH} / \mathrm{HF}$ ratio at baseline may not be eligible for cognitively focused behavioral interventions. However, that sample had a mean age of 41 years (intervention group) and symptom duration was not reported, which could affect ANS function in IBS patients. Jarrett et $\mathrm{al}^{38}$ reported no difference in the HRV patterns between IBS patients and healthy controls in a study of 7 to 10 yearold children in contrast to previous studies of adults. ${ }^{3,23,36}$ They suggested that age and symptom duration could affect ANS function in IBS patients. Likewise, we anticipated that ANS dysfunction could be reversed with CBT in a young population because the duration of dysfunction is shorter, and the associated physiologic changes may not be severe. Thus, based on our sample of young females with mean age of 21 , ANS dysfunction such as sympathetic hyperactivity and lower vagal tone may be reversible, and CBT can improve ANS function. Furthermore, the magnitude of the increase in the $\mathrm{HF}$ power and decrease in the $\mathrm{LF} / \mathrm{HF}$ ratio significantly 
correlated with the magnitude of the changes in GI symptoms. These findings clearly suggest that HRV is a significant potential objective marker for assessing ANS activities that are linked to the regulation of GI symptoms in young female patients with IBS-C.

In addition to GI symptom improvement, we found that CBT improved psychological symptoms, such as anxiety, depression, and stress in young female nursing students with IBS-C. Deechakawan et $\mathrm{al}^{39}$ reported that a cognitively focused behavioral intervention group had significantly lower levels of anxiety and depression at 6 and 12 months follow-up than the usual care group. However, in a study by Zernicke et al ${ }^{40}$ stress levels showed a significant rebound effect in the intervention group from post-intervention to 6-month follow-up even if stress levels were reduced in the cognitively focused behavioral intervention group. Moreover, in a study by Chilcot and Moss-Morris, ${ }^{41}$ anxiety and depression levels were similar between the CBT group and usual care group with a follow-up period of 6 months. The discrepancies in the previous findings may be attributed to differences in patients' age, sex, education level, and occupation. One of the reasons for the positive effects of CBT seen in our study may be the young age of the sample of female nursing students with IBS, who were willing to be active participants in the therapeutic group sessions and to share their thoughts, emotions, and behavior with their peers. Furthermore, changes in anxiety, depression, and stress correlated with changes in HF and LF/ HF over 6 months. Jarrett et $\mathrm{al}^{42}$ suggested anxiety and depressive disorders were related to decreased parasympathetic activity, both in women with IBS and control groups. Dishman et $\mathrm{al}^{43}$ reported that perceived emotional stress during the preceding week tended to decrease parasympathetic nervous system tone in healthy adults. Our results indicate that CBT could ameliorate ANS dysfunction through concomitant improvements in emotional status and psychological functioning. It is also notable that these simultaneous effects on HRV and psychological functioning could be sustained for 6 months after the completion of CBT.

Overall, our findings can be interpreted in the context of a biopsychosocial model with psychological and emotional factors interacting with physiological phenomena and perceptions of symptoms. ${ }^{44}$ In patients with IBS, psychological distress (eg, anxiety, depression) is known to be associated with the onset and exacerbation of IBS symptoms. ${ }^{15,45,46}$ The pathogenesis of this association between psychological distress and IBS symptoms may be explained by the dysregulations of the brain-gut axis which connects cognitive and emotional centers in the CNS with visceral afferent sensation, intestinal motility and secretion of the digestive tract. ${ }^{39,47-49}$ Therefore, psychological interventions such as $\mathrm{CBT}$ can reduce the severity of GI symptoms while improving physical and psychological functions by reducing the negative impact of CNS activity on gut function in patients with IBS. ${ }^{15,22,47,50}$

IBS symptom assessment is very important for determining the efficacy of certain treatment in IBS. However, severe refractory symptoms may preclude clinical decision making. In such cases, diagnostic tests such as endoscopy should not be delayed because IBS symptoms often overlap with more serious organic diseases, such as inflammatory bowel disease and gastrointestinal cancer. In contrast, patients with severe IBS symptoms in the absence of organic disease could be subjected to unnecessary repeated tests. ${ }^{13,51}$ Therefore, more reliable objective biomarkers are needed to supplement pathophysiologic disease status to assess the efficacy of certain therapies and avoid unnecessary invasive tests with unintended procedurerelated adverse effects. Objective biomarkers have been evaluated based on pathophysiologic mechanisms of IBS. One of the main mechanisms is the dysfunction of the brain-gut axis, particularly the ANS. HRV is a noninvasive, relatively easy and cost-effective method to evaluate ANS function indirectly. Whether changes in HRV are specific to IBS-C patients has not been established; that is, HRV itself cannot be generalized as a biomarker for IBS-C. However, HRV may be used to assess patients' response to CBT. For example, if the HRV pattern changes from a lower to higher HF power, somatic symptoms of IBS-C patients may be improved through continuing $\mathrm{CBT}$.

The current study has several limitations. First, IBS patients assigned to CBT may have had a placebo effect, which is particularly applicable to certain conditions for which symptoms are subjective and chronic, such as IBS. ${ }^{52}$ Owing to the nature of the intervention, participants may have known they were assigned to the treatment group and expected positive results. To reduce treatment bias, a third-party researcher who was blinded to group assignments monitored symptoms and HRV. Second, it included no healthy participants to compare the HRV responses with those of the intervention group. In future studies, healthy participants should be included to validate $H R V$ as a biomarker for distinguishing and treating patients with IBS. Third, all participants were females to control for sex-based differences in coping styles, levels of somatic and psychological symptoms, and physiological responses. ${ }^{8,9,15}$ Thus, the findings may not be applicable to males or middle-aged and older populations with IBS-C. Fourth, we focused on only IBS-C because of its more prominent autonomic dysfunction among the IBS types. $^{12}$ Therefore, the findings are specific to IBS-C at this point. Future studies should identify changes in the ANS in IBS subtyped by the predominant stool pattern for the efficacy of $\mathrm{CBT}$ to 
demonstrate the clinical utility of HRV as a reliable indicator. Last, based on the Rome III criteria, the GI symptoms of the participants in this study were not severe. Inclusion of a larger sample size and participants with more severe GI symptoms in a future study will provide more power to identify correlations between the role of the ANS and the efficacy of CBT in IBS patients to improve physical and psychological functioning.

In conclusion, CBT can increase $\mathrm{HF}$ power and decrease the $\mathrm{LF} / \mathrm{HF}$ ratio, resulting in the improvement of ANS activities with a close correlation to GI symptoms in young female patients with IBS-C. Indirect measurement of ANS activity using HRV may be a useful objective parameter for assessing the response to $\mathrm{CBT}$ in young female patients with IBS-C. However, the causal relationship between HRV and other behavioral outcomes should be investigated in a larger sample including both sexes and other subtypes of IBS.

Acknowledgements: We thank and remember Duk-Hee Kang for invaluable support throughout the study.

\section{Financial support: None.}

\section{Conflicts of interest: None.}

Author contributions: Conceived, designed, and planned study: Aelee Jang and Sun-Kyung Hwang; substantial contributions to data collection and conduct of study: Aelee Jang and SunKyung Hwang; data analysis: Aelee Jang and Nikhil S Padhye; and interpretation of results and manuscript preparation: Aelee Jang, Nikhil S Padhye, and Janet C Meininger. All authors approved the final draft of the manuscript.

\section{References}

1. Heidelbaugh JJ, Stelwagon M, Miller SA, Shea EP, Chey WD. The spectrum of constipation-predominant irritable bowel syndrome and chronic idiopathic constipation: US survey assessing symptoms, care seeking, and disease burden. Am J Gastroenterol 2015;110:580-587.

2. Brandt LJ, Chey WD, Foxx-Orenstein AE, et al. An evidence-based position statement on the management of irritable bowel syndrome. Am J Gastroenterol 2009;104(suppl 1):S1-S35.

3. Mazur M, Furgata A, Jabłoński K, Mach T, Thor P. Autonomic nervous system activity in constipation-predominant irritable bowel syndrome patients. Med Sci Monit 2012;18:CR493-CR499.

4. Lovell RM, Ford AC. Global prevalence of and risk factors for irritable bowel syndrome: a meta-analysis. Clin Gastroenterol Hepatol 2012;10:712-721, e4.

5. Mazurak N, Seredyuk N, Sauer H, Teufel M, Enck P. Heart rate vari- ability in the irritable bowel syndrome: review of the literature. Neurogastroenterol Motil 2012;24:206-216.

6. Bensoussan A, Kellow JE, Bourchier SJ, et al. Efficacy of a Chinese herbal medicine in providing adequate relief of constipation-predominant irritable bowel syndrome: a randomized controlled trial. Clin Gastroenterol Hepatol 2015;13:1946-1954, e1.

7. Quigley E, Fried M, Gwee KA, et al. Practice guideline, irritable bowel syndrome: a global perspective. Arab J Gastroenterol 2010;11:53-62.

8. Si JM, Wang LJ, Chen SJ, Sun LM, Dai N. Irritable bowel syndrome consulters in Zhejiang province: the symptoms pattern, predominant bowel habit subgroups and quality of life. World J Gastroenterol 2004;10:1059-1064

9. Lee OY, Mayer EA, Schmulson M, Chang L, Naliboff B. Genderrelated differences in IBS symptoms. Am J Gastroenterol 2001;96:21842193.

10. Nellesen D, Yee K, Chawla A, Lewis BE, Carson RT. A systematic review of the economic and humanistic burden of illness in irritable bowel syndrome and chronic constipation. J Manag Care Pharm 2013;19:755-764.

11. Harris L. Irritable bowel syndrome - advances in diagnosis, pathophysiology, and treatment. Business Briefing: Women's Healthcare 2004;8997.

12. Yildirim AE, Korkmaz M, Altun R, Sandikçi SC, Ocal S, Selçuk H. Is there any association between irritable bowel syndrome subgroups and autonomous dysfunction. Eur Rev Med Pharmacol Sci 2016;20:13151322.

13. Plavšić I, Hauser G, Tkalčić M, Pletikosić S, Salkić N. Diagnosis of irritable bowel Syndrome: role of potential biomarkers. Gastroenterol Res Pract 2015;2015:490183.

14. Pellissier S, Dantzer C, Canini F, Mathieu N, Bonaz B. Psychological adjustment and autonomic disturbances in inflammatory bowel diseases and irritable bowel syndrome. Psychoneuroendocrinology 2010;35:653662.

15. Deechakawan W, Cain KC, Jarrett ME, Burr RL, Heitkemper MM. Effect of self-management intervention on cortisol and daily stress levels in irritable bowel syndrome. Biol Res Nurs 2013;15:26-36.

16. Adeyemi EO, Desai KD, Towsey M, Ghista D. Characterization of autonomic dysfunction in patient with irritable bowel syndrome by means of heart rate variability studies. Am J Gastroenterol 1999;94:816-823.

17. Salvioli B, Pellegatta G, Malacarne M, et al. Autonomic nervous system dysregulation in irritable bowel syndrome. Neurogastroenterol Motil 2015;27:423-430

18. Heitkemper M, Burr RL, Jarrett M, Hertig V, Lustyk MK, Bond EF. Evidence for autonomic nervous system imbalance in women with irritable bowel syndrome. Dig Dis Sci 1998;43:2093-2098.

19. Schmulson M, Lee OY, Chang L, Naliboff B, Mayer EA. Symptom differences in moderate to severe IBS patients based on predominant bowel habit. Am J Gastroenterol 1999;94:2929-2935.

20. Heitkemper M, Jarrett M, Cain KC, et al. Autonomic nervous system function in women with irritable bowel syndrome. Dig Dis Sci 2001;46:1276-1284

21. Liu Q, Wang EM, Yan XJ, Chen SL. Autonomic functioning in irritable bowel syndrome measured by heart rate variability: a meta-analysis. J Dig 
Dis 2013;14:638-646.

22. Boyce PM, Talley NJ, Balaam B, Koloski NA, Truman G. A randomized controlled trial of cognitive behavior therapy, relaxation training, and routine clinical care for the irritable bowel syndrome. Am J Gastroenterol 2003;98:2209-2218

23. Jarrett ME, Cain KC, Barney PG, et al. Balance of autonomic nervous system predicts who benefits from a self-management intervention program for irritable bowel syndrome. J Neurogastroenterol Motil 2016;22:102-111.

24. Jang AL, Hwang S, Kim DU. The effects of cognitive behavioral therapy in female nursing students with irritable bowel syndrome: a randomized trial. Eur J Gastroenterol Hepatol 2014;26:918-926.

25. Park JM, Choi MG, Cho YK, et al. Functional gastrointestinal disorders diagnosed by Rome III Questionnaire in Korea. J Neurogastroenterol Motil 2011;17:279-286.

26. Faul F, Erdfelder E, Buchner A, Lang AG. Statistical power analyses using $G^{\star}$ Power 3.1: Tests for correlation and regression analyses. Behav Res Methods 2009;41:1149-1160.

27. Berntson GG, Bigger JT Jr, Eckberg DL, et al. Heart rate variability: origins, methods, and interpretive caveats. Psychophysiology 1997;34:623648.

28. Malliani A, Pagani M, Lombardi F, Cerutti S. Cardiovascular neural regulation explored in the frequency domain. Circulation 1991;84:482492.

29. Heseltine D, Potter JF, Hartley G, Macdonald IA, James OF. Blood pressure, heart rate and neuroendocrine responses to a high carbohydrate and a high fat meal in healthy young subjects. Clin Sci (Lond) 1990;79:517-522.

30. Wiklund IK, Fullerton S, Hawkey CJ, et al. An irritable bowel syndrome-specific symptom questionnaire: development and validation. Scand J Gastroenterol 2003;38:947-954.

31. Dimenäs E, Glise H, Hallerbäck B, Hernqvist H, Svedlund J, Wiklund I. Well-being and gastrointestinal symptoms among patients referred to endoscopy owing to suspected duodenal ulcer. Scand J Gastroenterol 1995;30:1046-1052.

32. Zigmond AS, Snaith RP. The hospital anxiety and depression scale. Acta Psychiatr Scand 1983;67:361-370.

33. Oh SM, Min KJ, Park DB. [A study on the standardization of the hospital anxiety and depression scale for Koreans: a comparison of normal, depressed and anxious groups.] J Korean Neuropsychiatr Assoc 1999;38:289-296. [Korean]

34. Linn MW. A global assessment of recent stress (GARS) scale. Int J Psychiatry Med 1985-1986;15:47-59.

35. Koh KB, Park JK. [Validity and reliability of the Korean version of the global assessment of recent stress scale.] Korean J Psychosom Med 2000;8:201-211. [Korean]

36. Cain KC, Jarrett ME, Burr RL, Hertig VL, Heitkemper MM. Heart rate variability is related to pain severity and predominant bowel pattern in women with irritable bowel syndrome. Neurogastroenterol Motil
2007;19:110-118.

37. Saha L. Irritable bowel syndrome: pathogenesis, diagnosis, treatment, and evidence-based medicine. World J Gastroenterol 2014;14;20:67596773.

38. Jarrett M, Heitkemper M, Czyzewski D, Zeltzer L, Shulman RJ. Autonomic nervous system function in young children with functional abdominal pain or irritable bowel syndrome. J Pain 2012;13:477-484.

39. Deechakawan W, Heitkemper MM, Cain KC, Burr RL, Jarrett ME. Anxiety, depression, and catecholamine levels after self-management intervention in irritable bowel syndrome. Gastroenterol Nurs 2014;37:24-32.

40. Zernicke KA, Campbell TS, Blustein PK, et al. Mindfulness-based stress reduction for the treatment of irritable bowel syndrome symptoms: a randomized wait-list controlled trial. Int J Behav Med 2013;20:385-396.

41. Chilcot J, Moss-Morris R. Changes in illness-related cognitions rather than distress mediate improvements in irritable bowel syndrome (IBS) symptoms and disability following a brief cognitive behavioural therapy intervention. Behav Res Ther 2013;51:690-695.

42. Jarrett ME, Burr RL, Cain KC, Hertig V, Weisman P, Heitkemper MM. Anxiety and depression are related to autonomic nervous system function in women with irritable bowel syndrome. Dig Dis Sci 2003;48:386-394.

43. Dishman RK, Nakamura Y, Garcia ME, Thompson RW, Dunn AL, Blair SN. Heart rate variability, trait anxiety, and perceived stress among physically fit men and women. Int J Psychophysiol 2000;37:121-133.

44. Drossman DA. Presidential address: gastrointestinal illness and the biopsychosocial model. Psychosom Med 1998;60:258-267.

45. Drossman DA, Creed FH, Olden KW, Svedlund J, Toner BB, Whitehead WE. Psychosocial aspects of the functional gastrointestinal disorders. Gut 1999;45(suppl 2):II25-II30.

46. Hertig VL, Cain KC, Jarrett ME, Burr RL, Heitkemper MM. Daily stress and gastrointestinal symptoms in women with irritable bowel syndrome. Nurs Res 2007;56:399-406.

47. Drossman DA, Corazziari E, Talley NJ, Thompson WG, Whitehead WE. Rome II. The functional gastrointestinal disorders - diagnosis, pathophysiology and treatment: a multinational consensus. 2nd edn. McLean, Va: Degnon Associates; 2000:355-375.

48. Jones M, Koloski N, Boyce P, Talley NJ. Pathways connecting cognitive behavioral therapy and change in bowel symptoms of IBS. J Psychosom Res 2011;70:278-285.

49. Posserud I, Ersryd A, Simrén M. Functional findings in irritable bowel syndrome. World J Gastroenterol 2006;12:2830-2838.

50. Labus J, Gupta A, Gill HK, et al. Randomised clinical trial: symptoms of the irritable bowel syndrome are improved by a psycho-education group intervention. Aliment Pharmacol Ther 2013;37:304-315.

51. Quigley EM, Clarke G. A serologic test for irritable bowel syndrome: real meat or bare bones? Gastroenterology 2009;137:2168-2170.

52. Cuijpers P, Karyotaki E, Andersson G, Li J, Mergl R, Hegerl U. The effects of blinding on the outcomes of psychotherapy and pharmacotherapy for adult depression: a meta-analysis. Eur Psychiatry 2015;30:685-693. 